\title{
Application testing of a new three-dimensional acceleration measuring system with wireless data transfer (WAS) for behavior analysis
}

\author{
KLAUS MANFRED SCHEIBE and CORA GROMANN \\ Institute for Zoo and Wildlife Research, Berlin, Germany
}

\begin{abstract}
A wireless acceleration measurement system was applied to free-moving cows and horses. Sensors were available as a collar and a flat box for measuring leg or trunk movements. Results were transmitted simultaneously by radio or stored in an 8-MB internal memory. As analytical procedures, frequency distributions with standard deviations, spectral analyses, and fractal analyses were applied. By means of the collar sensor, basic behavior patterns (standing, grazing, walking, ruminating, drinking, and hay uptake) could be identified in cows. Lameness could be detected in cows and horses by means of the leg sensor. The portion of basic and harmonic spectral components was reduced; the fractal dimension was reduced. The system can be used for the detection and analysis of even small movements of free-moving humans or animals over several hours. It is convenient for the analysis of basic behaviors, emotional reactions, or events causing flight or fright or for comparing different housing elements, such as floors or fences.
\end{abstract}

Movements of animal bodies or single extremities often have been measured by optical methods (Falaturi, 2001; Szalay, Back, Barneveld, Schamhardt, \& Hajos, 2002). Such procedures require a stationary recording place, and three-dimensional movement recordings can be obtained only under certain circumstances. A three-dimensional acceleration measurement system with a wire connection has been offered for technical, as well as for biological, applications (Xsens MT9; see Xsens Technologies, 2003). It has been applied to motion analysis in humans (Keshner, 2004; Mathie, Coster, Lovell, \& Celler, 2004). Systems with two-dimensional acceleration measurements are not sufficient for a complete description of spatial movements, since the resulting force cannot be obtained from only two axes.

Analyzing the movements of free-moving animals requires small sensors and the storage of large data sets or wireless data transmission. Basic behaviors (walking, running, or grazing) typically involve movements of the whole body. They are characterized by body posture, characteristic movement directions, and distinct movement frequencies. Accordingly, it should be possible to identify different behaviors or movement patterns by analyzing the complete acceleration pattern from a single point on

The investigation was supported by the PRO INNO program of the German Federal Ministry of Business and Technology, KF 0331701KRF1. We further acknowledge the support of Dr. Lebelt, Horse Clinic Havelland, the Cattle Clinic of the Free University Berlin, Mrs. A. Wessel, Trappenfelde, and Mr. A. Broja, Liebenthal. Correspondence concerning this article should be addressed to K. M. Scheibe, Institute for Zoo and Wildlife Research, P.O. Box 601103, D-10252, Berlin, Germany (e-mail: kscheibe@izw-berlin.de). the body also. A sensor system for this purpose should be small and lightweight, but also shock resistant and simple to affix to the animal's body. By means of such sensors, it should be possible to identify not only basic behaviors, but also deviations from normal movement patterns resulting from diseases or external disturbance.

\section{Animals, Material, and Methods}

A three-dimensional wireless acceleration measurement system (WAS) has been tested in two versions (GreenWay Systeme, 2004). The instrument measures accelerations for the three spatial axes in the range of $\pm 2 \mathrm{~g}$ linearly, up to $\pm 4 \mathrm{~g}$ with a damped characteristic. A version for $\pm 10 \mathrm{~g}$ can also be obtained. For recording, sample rates of 10,100 , and $1,000 \mathrm{msec}$ can be selected. In all the experiments described here, a sample rate of $10 \mathrm{msec}$ was applied. Data can be transmitted simultaneously by a radio link and displayed on a laptop or a PC. The radio communication works over a distance of up to $200 \mathrm{~m}$ on a free range and $50 \mathrm{~m}$ in inconvenient conditions. Alternatively, the data can be stored internally in an 8-MB memory and transmitted later on command. Since communication is possible only to one sensor at a time, the storage option enables the application of several sensors simultaneously. The results are stored in EXCEL-compatible text files containing the time and the three simultaneously recorded acceleration values. Two different sensors were applied: a collar sensor and a flat box for fixation at the extremities or the trunk. Data were recorded from 4 cows (1 on the free range and 3 at the veterinary clinic of the Free University of Berlin), from a horse at the Horse Clinic Havelland, and from 2 horses at a riding place. The collar sensor was applied in experiments in which basic behaviors of a cow 
on a free range were identified. The behaviors were simultaneously recorded visually. From measurements made over several hours, samples 10 and $5 \mathrm{sec}$ in duration from the middle of periods of constant behaviors were selected and analyzed.

The flat box sensor was fixed by means of horse boots at the legs of the cows and the horses. The animals were led by the animal keepers at different velocities on concrete. In some cases, the collar sensor was simultaneously applied. The results were analyzed in order to identify the different movement patterns of stepping, trotting, and galloping (horses) and to distinguish healthy from lame animals. The data basis selected for analysis consisted of 155 samples of 1,000 values (for frequency distributions) and of 500 values (for all the other procedures).

All analysis was done with a methodical background in order to test the equipment, to develop different analytical procedures, and to exemplify the value of the approach. It was not our aim to conduct a systematical comparative study, for example, of disease diagnosis.

Several mathematical procedures were developed for the evaluation of movements. At first, for each data point, the resulting force was computed from the three acceleration values.

In a second step, frequency distributions were determined from 10-sec samples for the three axes and for the resulting force separately.

From the time series of the resulting force, the fractal dimension $D$ was determined (Ripoli et al., 1995). From the same time series, the power spectrum was also computed.

The total power, the power of the basic frequency, the total power of the harmonic components, and the total

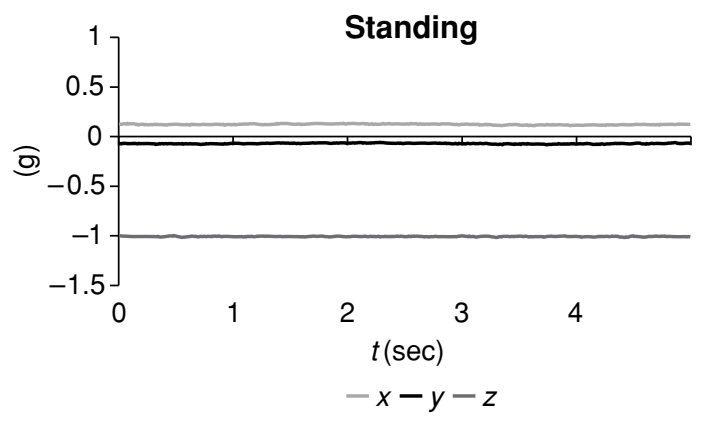

Grazing

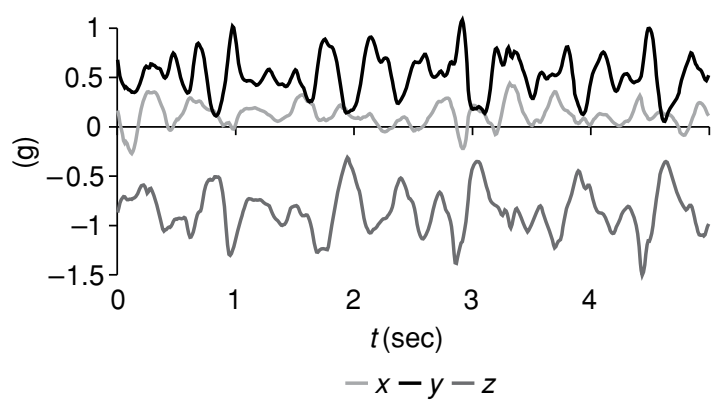

power of the nonharmonic components were determined from the power spectra.

In addition to absolute acceleration values, derived complex characteristics of movements, such as linearity or the relation between the harmonic and the nonharmonic components, were obtained and compared.

The experiments had three different aims: first, to test the recording device under realistic field conditions; second, to find analytical methods for potential automatic behavior identification on the basis of three-dimensional acceleration recordings; and third, to find out whether disturbed movement patterns, such as lameness, can be identified by three-dimensional acceleration recordings in field conditions. Our basic assumption was not only that absolute accelerations should be regarded, but also that complex analyses of time-dependent acceleration patterns are required.

\section{Results}

Identification of behavior patterns in cattle by the collar sensor. Already, by a visual inspection of the three-axial acceleration patterns, basic behaviors such as standing, walking, grazing, or rumination can be identified. The gravitation enables the identification of the sensors' position and, accordingly, the position of the head (Figure 1).

Grazing is characterized by a lowered head, whereas rumination occurs with the head up, which can be recognized by the different acceleration values along the $y$-axis. The frequency distributions of the acceleration values during the different behaviors are approximately normally distributed but differ in deviation and mean value (Fig-

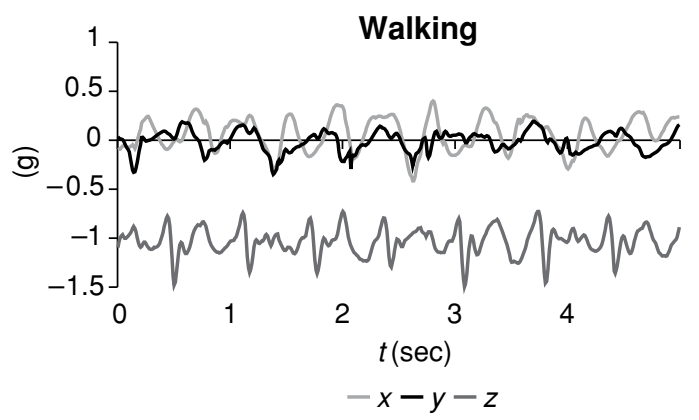

Ruminating

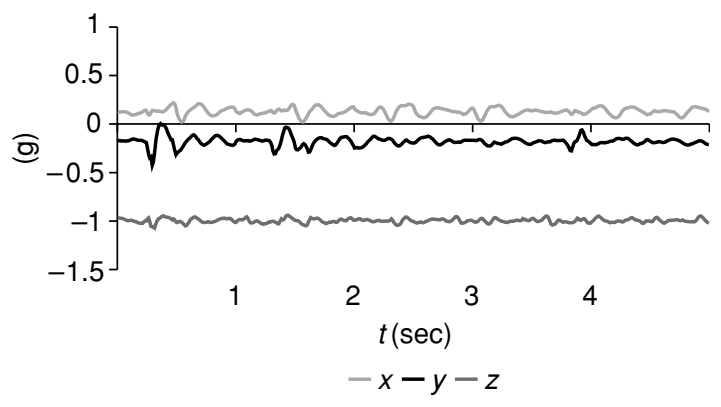

Figure 1. Original acceleration patterns from a cow during different behaviors (collar). 


\section{Standing}
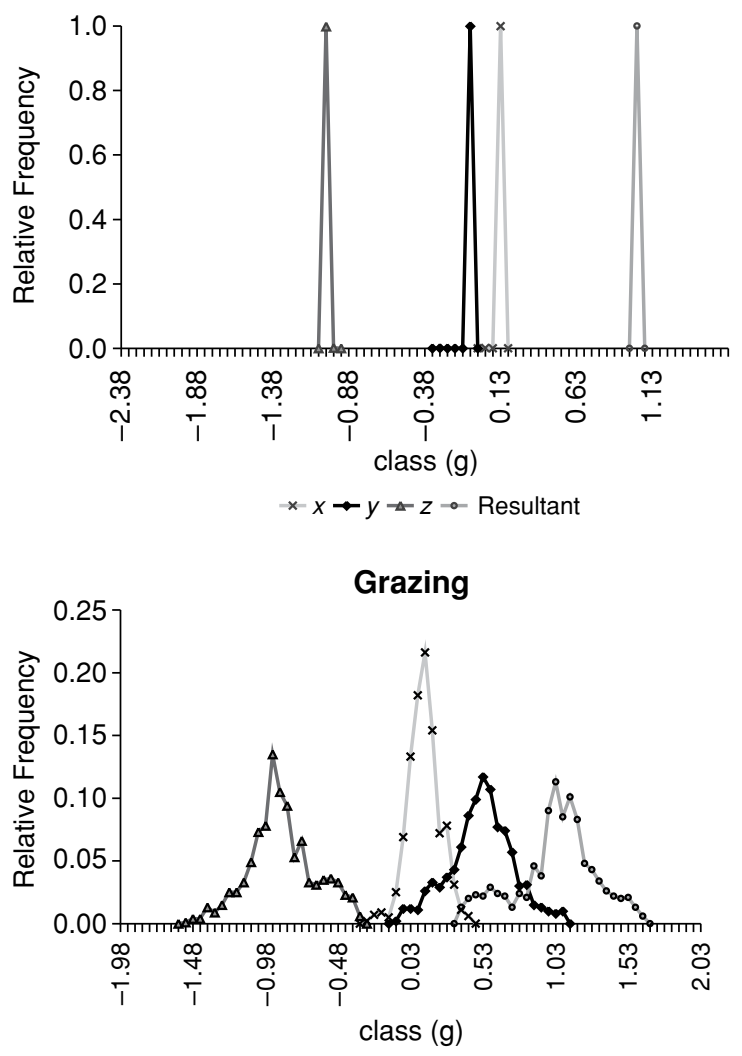

$x x \rightarrow y-z=-$ Resultant
Walking

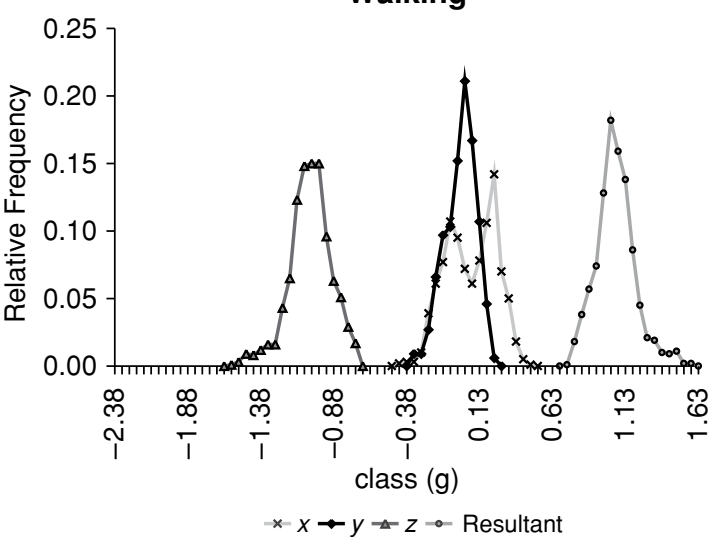

Ruminating

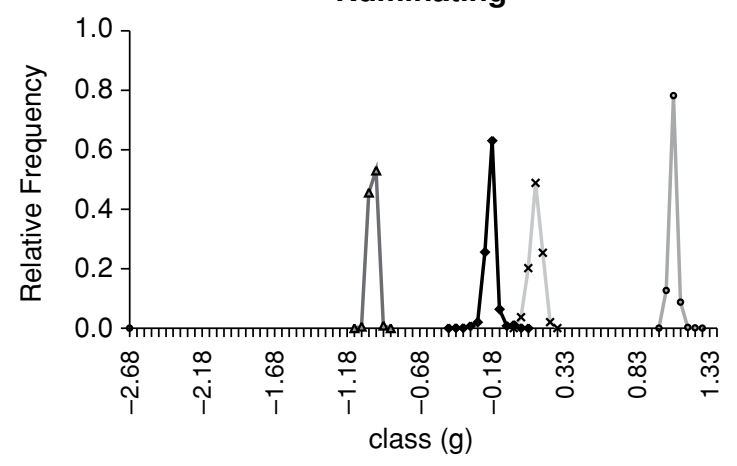

$x x \rightarrow y-\Delta-z \rightarrow$ Resultant

Figure 2. Frequency distributions of accelerations during different behaviors.

ures 2 and 3). Grazing can be distinguished from all other behaviors by the different mean of the $y$-accelerations in the frequency distributions. On the basis of the standard deviations alone, hay uptake, grazing, and rumination can be clearly distinguished, but the values for walking and hay uptake overlap. Rumination can be identified automatically by a self-developed procedure based on the standard deviation.

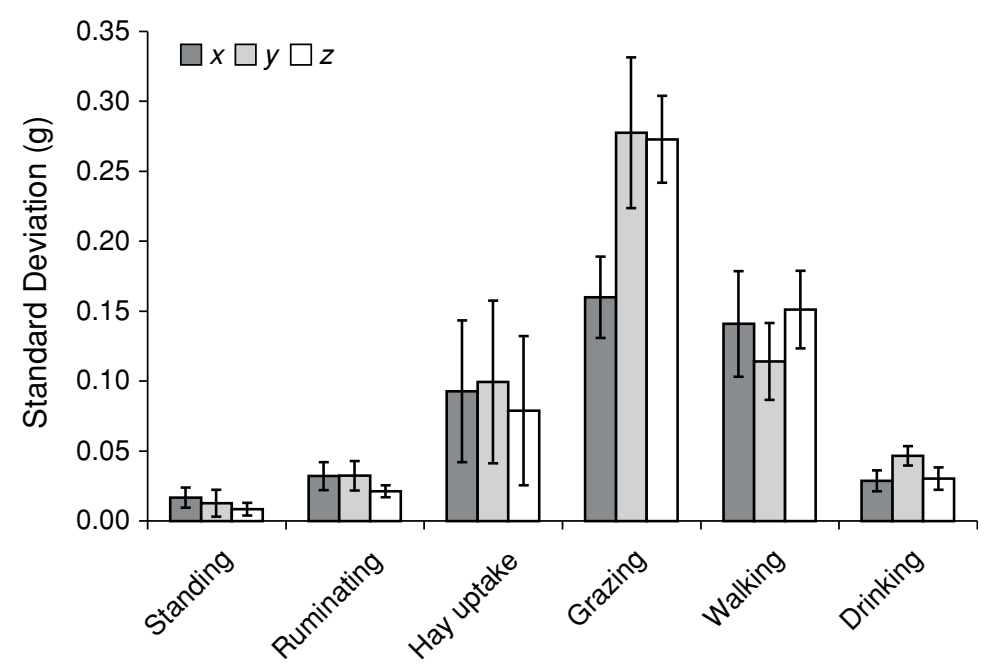

Figure 3. Standard deviations of acceleration values during different behaviors. 

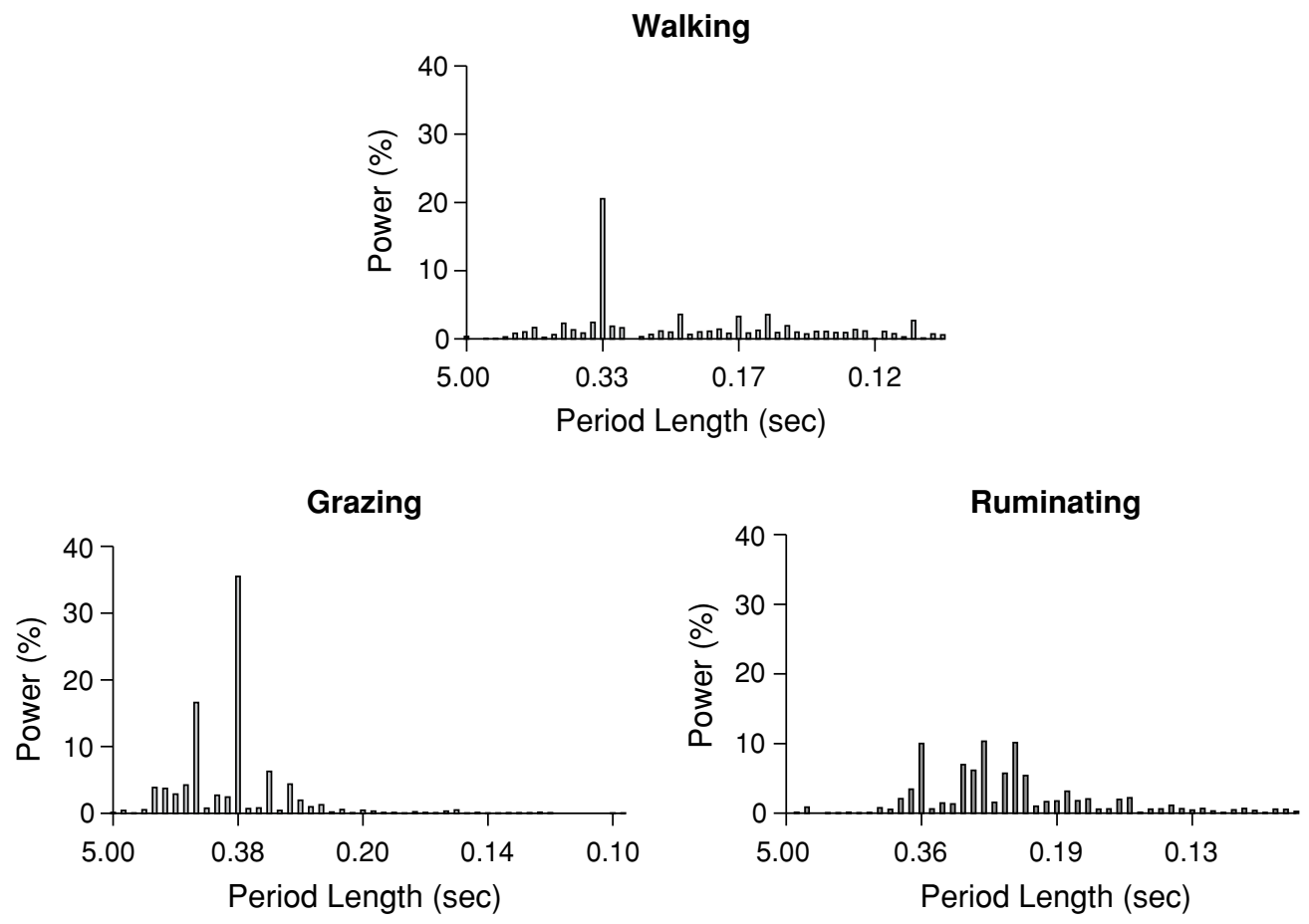

Figure 4. Power spectra of accelerations during different behaviors.

The spectral components of time series of $5 \mathrm{sec}$ were determined by Fourier analysis. From the original resulting values, the autocorrelation function was computed to reduce the noise level; thereafter, the autocorrelation was analyzed for cosine functions, and the portions of the different periods were determined. These portions were displayed as power spectra.
The grazing, walking, and ruminating behaviors result in regular movements of the head and neck. Accordingly, single different periods of different period lengths can be detected from the collar sensor during these behaviors (Figure 4).

Also, the fractal dimension can be used to distinguish some behaviors, but the deviations overlap between walk-

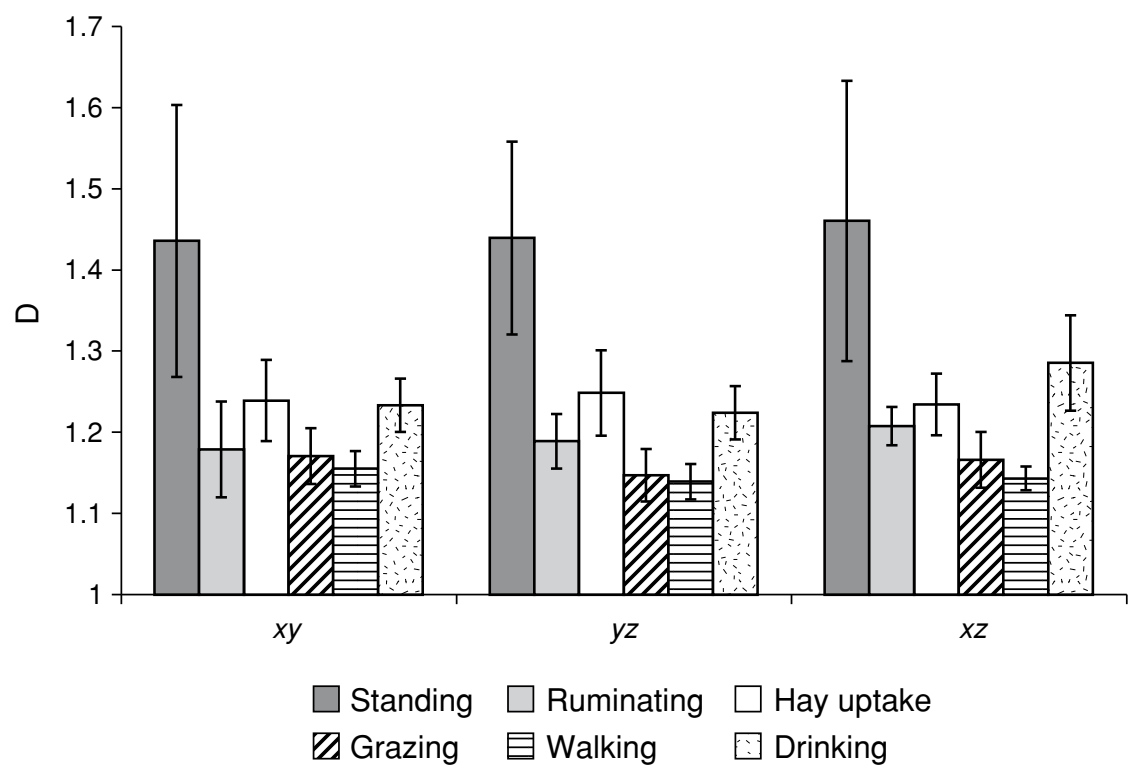

Figure 5. Fractal dimension of 2-D acceleration patterns in the three spatial planes during different behaviors. 


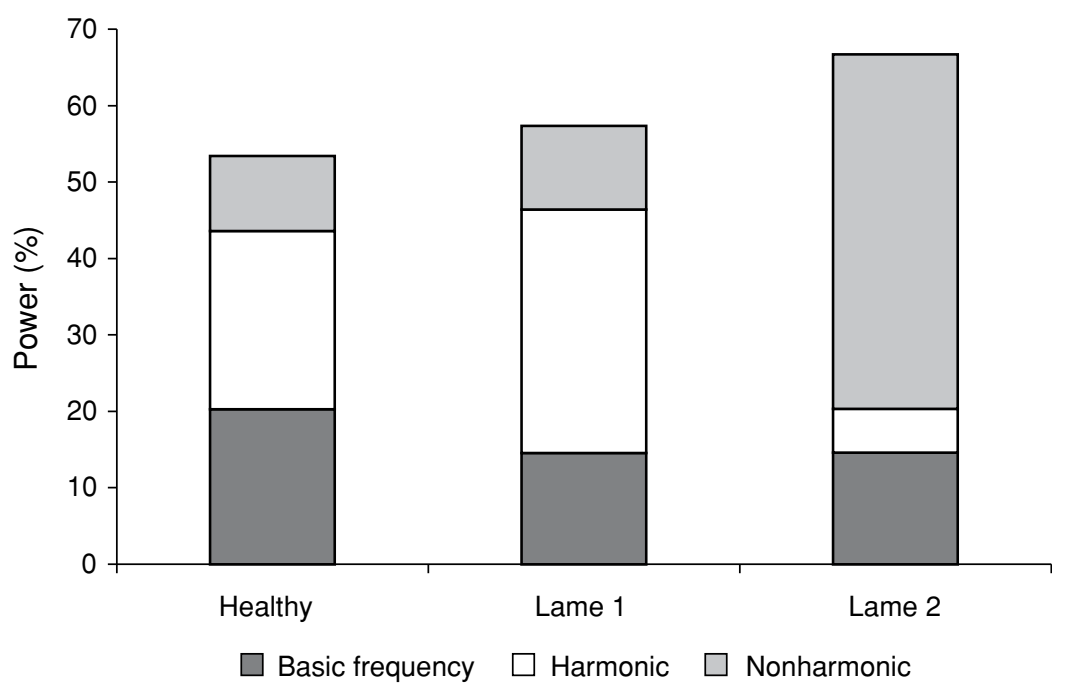

Figure 6. Classification of power spectra in three cows.

ing and grazing. The lowest overlaps can be seen in the $x-z$ plane. Only rumination and hay uptake can be better distinguished by the fractal dimension in the other planes (Figure 5).

Recognition of lameness. The original acceleration patterns during walking differed only slightly between healthy and lame cows. Much more clear differences were detectable on the basis of the power spectra of the resulting forces. In the 2 diseased cows, the power of the basic frequency was lowered, and the other frequencies were predominant (basic frequency was $20.25 \%$ in the healthy cow vs. $14.52 \%$ and $14.56 \%$ in the lame individuals). The nonharmonic components were highly elevated in Cow 2 (Figure 6). This individual obviously had a higher degree of lameness than did Cow 1.
In the lame cows, the fractal dimensions of the leg movements were substantially lower than those in the healthy individual. This indicates a higher degree of linearity in the movements of the diseased animals (Figure 7).

The spectral analysis gave a similar result in horses, in comparison with the cows. The harmonic components were as high as $97 \%$ in the healthy animals. In an only slightly lame animal, 49\% harmonic components were found if it was walking while being led and $42 \%$ if it ran alone. After a provocation test, the harmonic components were lowered to only $5 \%$. Nonharmonic components were hardly found in the healthy animals. The relation between basic frequencies and harmonic and nonharmonic components indicated a lowered expression of the basic frequency in the diseased animal (Figure 8).

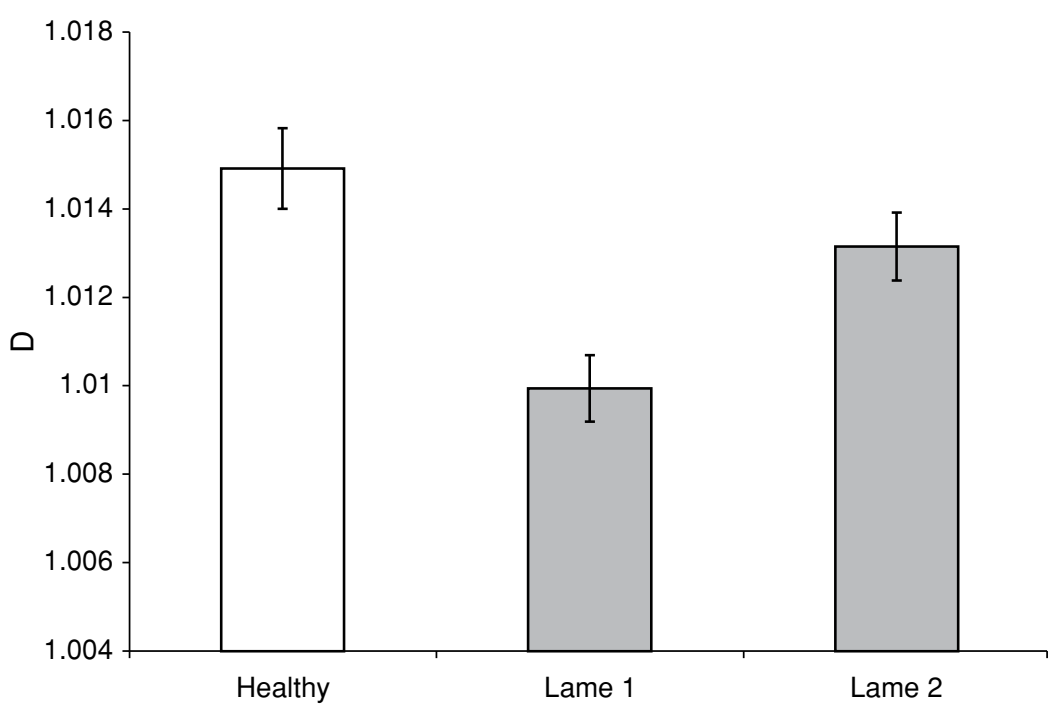

Figure 7. Fractal dimensions of acceleration patterns in three cows. 


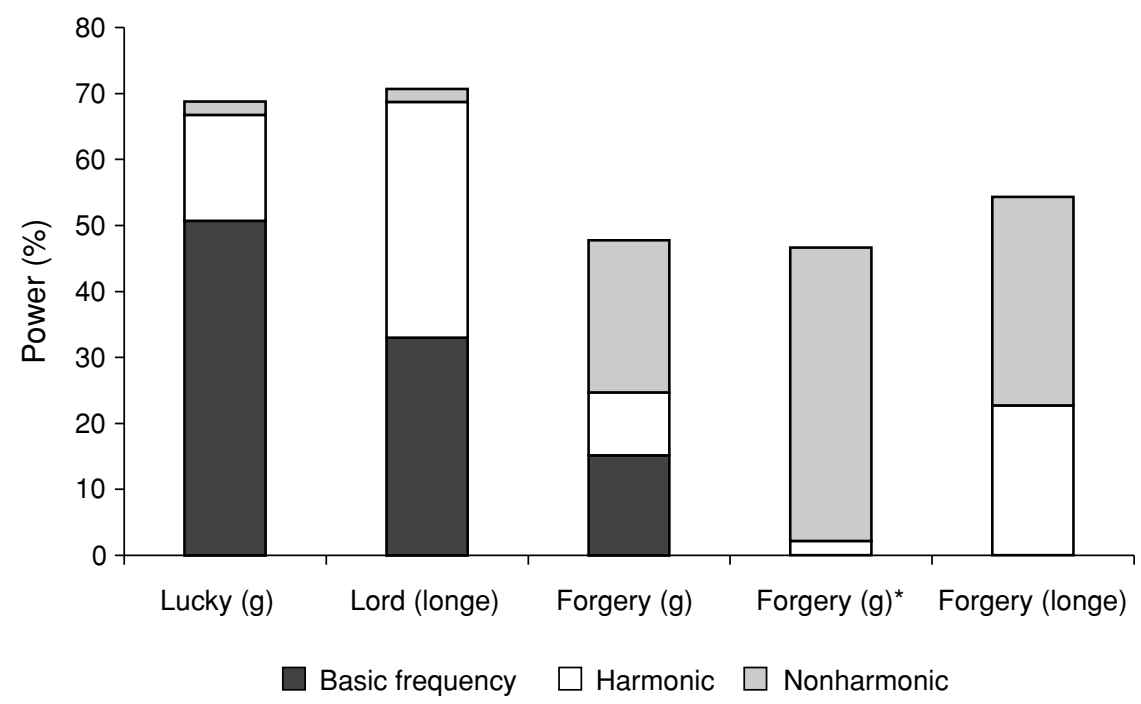

Figure 8. Classification of power spectra from horses ("Forgery" with slight signs of lameness, *after provocation).

The fractal dimension was higher during stepping in healthy cows and healthy horses, in comparison with diseased individuals of both species. A difference was not recognizable during trotting and galloping (Figure 9).

\section{Discussion}

The three-dimensional acceleration measurement system was sufficiently sensible and, as such, very convenient for recording movements of free-moving cattle and horses. We never observed aversive reactions of the animals against the sensors or their application. The measuring range was sufficient for most cases; only heavy movements, such as jumping, caused overflows. Most normal behaviors of the animals recorded by the collar caused ac- celerations lower than $2 \mathrm{~g}$; only the leg sensor sometimes recorded higher accelerations. The damped characteristic with the possibility of recording accelerations of up to $4 \mathrm{~g}$ also enabled us, in this case, to record an acceleration curve and to compare curves from different animals or situations. For research on highly intensive movements, such as jumping, the version with the measuring range of $10 \mathrm{~g}$ may be recommended. The data transmission worked highly reliably on distances of more than $100 \mathrm{~m}$ in all situations. With this simultaneous data transmission, the acceleration curves could be followed directly on the screen. The storage mode made it possible to apply two sensors at the same time on one and the same animal at the leg and the neck. The advantage of the system over two-

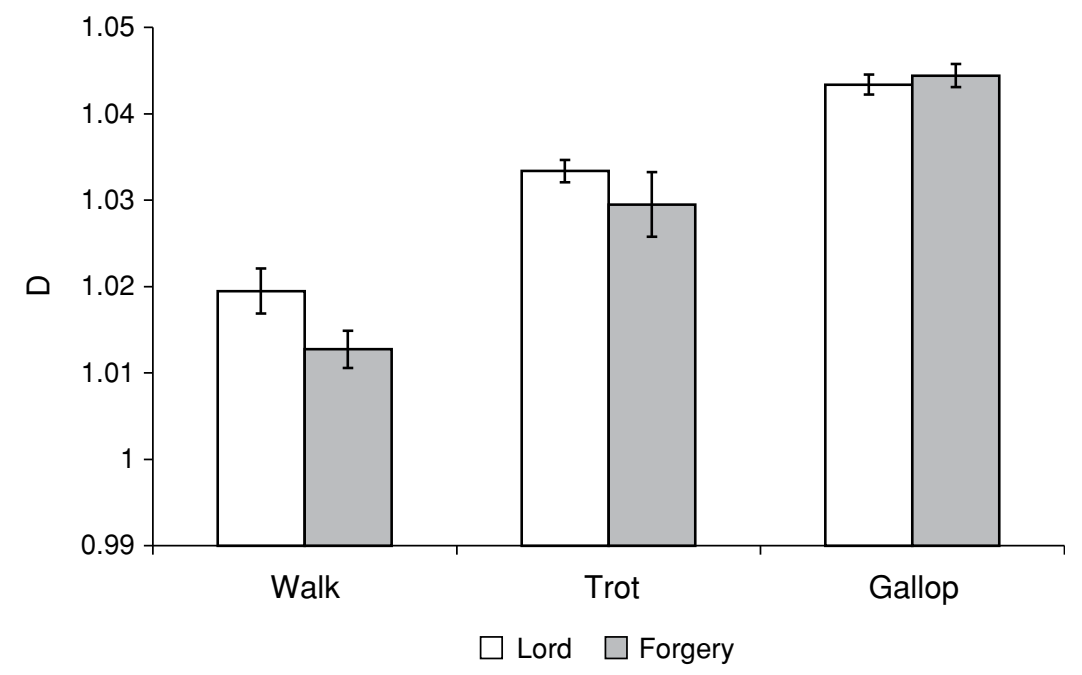

Figure 9. Fractal dimension of leg movements in horses measured at the right hind leg ("Forgery" with slight signs of lameness). 
dimensional recorders consists in offering the possibility of computing the resulting force. This can be done from free-moving individuals in very different situations. The application is restricted only by power consumption and battery capacity. The sensor has a time of independence of about $16 \mathrm{~h}$.

If the results from the different axes are compared, the influence of the gravitation gives information about the spatial orientation of the sensor and the parts of the animal's body. The head position gives valuable information for the detection of different behaviors. On the other hand, neither the position information nor absolute acceleration values (frequency distribution) give sufficient information to recognize all basic behaviors or disturbed behavior patterns. For the identification of behavior patterns, standard deviation and frequency spectra were useful procedures; in addition, the fractal dimension can be applied. A hierarchic classification procedure will be needed for the automatic identification of behaviors.

To identify lameness, movement analyses were done under strictly controlled conditions. A computerized analysis of the movements of a foreleg and the head was undertaken for horses on a walk belt by Schobesberger and Peham (2002). They applied a vibration analysis and a neural network and developed an automatic procedure for recognizing lameness. Such investigations require a great deal of effort, which could be substantially reduced by wireless acceleration measurements. The Equimétrix measuring system is a two-dimensional acceleration recorder. By means of it, dorsoventral and dorsolateral accelerations can be measured (Lindner, 1999). Nevertheless, the resulting force cannot be computed from these values. Against this background, the WAS offers the possibility of recording and analyzing movements from freely moving animals under field conditions. To recognize disturbed movement patterns, power spectral analysis was extremely valuable. The classification into basic frequency and harmonic and nonharmonic components gave reliable results in the two species.

The fractal dimension varied in the same direction between healthy and lame animals in both species. The higher fractal dimension in healthy animals can be interpreted as a sign of more complex movements, whereas in lame animals, linear components are more prevalent.
As a whole, the complex evaluation of movements by means of this wireless three-dimensional acceleration measuring system may be useful in many fields of research and is not restricted to the examples described. Such a measuring system and the different analytical methods could, for example, be applied in the investigation of emotional reactions or complex movements from animals, as well as from humans. It could also be used to record the basic behavior patterns of patients, in parallel with storage ECG records, or for research purposes with regard to reactions to external disturbances or nervosity. It could also be applied to the investigation of locomotor patterns on different floors, reactions to different housing equipments, and much more.

\section{REFERENCES}

Falaturi, P. H. (2001). Computerkinematographie (CKG) als geeignetes Verfahren zur objektiven Bewegungsanalyse: Beschreibung und Ergebnisse. Pferdeheilkunde, 17, 30-41.

GreenWay Systeme GmbH (2004). WAS-Wireless accelerometry system: Technical documentation. Frankfurt: Author.

Keshner, E. A. (2004). Head-trunk coordination in elderly subjects during linear anterior-posterior translations. Experimental Brain Research, 158, 213-222.

Lindner, A. (Ed.) (1999). Beiträge aus Orthopädie, Ganganalytik und Doping. Dortmund: Lensing.

Mathie M. J., Coster, A. C. F., Lovell, N. H., \& Celler, B. G. (2004). Accelerometry: Providing an integrated, practical method for long-term, ambulatory monitoring of human movement. Physiological Measurement, 25, R1-R20.

Ripoli, A., Palagi, G., Bedini, R., Rizzo, V., Kraft, G., \& Franchi, D. (1995). A fractal analysis approach to obtain effective algorithms usable in biotelemetry on-line signal analysis. In C. Cristalli, C. J. Amlaner, \& M. R. Neuman (Eds.), Proceedings of the 13th International Symposium on Biotelemetry (pp. 407-413). Terre Haute: Indiana State University Press.

Schobesberger, H., \& Peham, C. (2002). Computerized detection of supporting forelimb lameness in the horse using an artificial neural network. Veterinary Journal, 163, 77-84.

Szalay, F., Back, W., Barneveld, A., Schamhardt, H., \& Hajos, F. (2002). Comparison of VHS video recording system with Apple Macintosh-based image analysis and modified CODA-3 systems in equine motion analysis. Acta Veterinaria Hungarica, 50, 167-176.

Xsens TeChNOlogies (2003). Technical data: MT9 real-time 3D motion tracker. Enschede, The Netherlands: Author.

(Manuscript received September 27, 2005; revision accepted for publication January 21,2006 .) 